\title{
A NEW ANALYTICAL INVESTIGATION OF NATURAL CONVECTION OF NON- NEWTONIAN NANOFLUIDS FLOW BETWEEN TWO VERTICAL FLAT PLATES BY THE GENERALIZED DECOMPOSITION METHOD (GDM)
}

\author{
M.Kezzar ${ }^{* 1}$, N. Nafir ${ }^{1}$, I. Tabet ${ }^{1,{ }^{*},}$ A. Khanetout ${ }^{2}$
}

\begin{abstract}
In this work, natural convection in a non-Newtonian fluid/nanofluid between two vertical plates is investigated. The study was carried out on three types of nanofluids, namely Silver/Water, Oxide CopperWater and Titanium oxide/Water. The mathematical formulation gives a set of strongly coupled nonlinear ordinary differential equations of the second order. These equations, characterizing velocity and temperature distributions, were solved numerically by the Runge-Kutta fourth order method, and analytically by a new Adomian of decomposition approach named the Adomian generalized method (GDM). The results show clearly the effectiveness, accuracy and applicability of the used technique (GDM). Using nanoparticles (Ag, $\mathrm{C}_{\mathrm{uO}}$ and $\mathrm{TiO}_{2}$ ) in water as a base fluid substantially increases the coefficient of friction and characteristics of heat transfer. Compared to other works, the generalized Adomian decomposition technique (GDM) offers the advantages of precision and velocity of convergence.
\end{abstract}

Keywords: Naturel Convection, Nanofluids, Analytical Methods, Adomian Generalized Method (GDM)

\section{INTRODUCTION}

The natural convection of a non-Newtonian fluid / nanofluid between two vertical planar walls attracted the attention of researchers interested in the resolution of non-linear ordinary differential equations characterizing velocity and temperature profiles, either analytically or numerically. The studies conducted by Ostrach [1] and Khalifa [2] showed the effect and importance of non-Newtonian fluids on velocity and temperature profiles for new industrial applications. Jha \& al. [3] solved the problem of the natural convection of a viscous incompressible fluid between two infinite parallel vertical walls by using Laplace Transform method.

Noghrehabadi [4] attempted to characterize heat and mass transfer in the natural convection of a fluid on a vertical plate in a saturated porous medium by both a set of coupled non-linear ordinary differential equations, and by digital processing (finite difference method) to account for friction. Rashad [5] gives a theoretical view of the effect of the velocity of uniform perspiration on the natural convection flow of the boundary layer of a non-Newtonian fluid between two non-parallel vertical walls in a porous medium saturated by a nanofluid. The mathematical formulation of this problem gives a set of strongly coupled nonlinear equations that were solved numerically by the method of implicit finite difference. Finally, this study concludes with a comparison with previous works.

Narahar [6] succeeded in obtaining an exact solution to account for the natural convection flow of a viscous incompressible fluid between two vertical walls, in the presence of a source of thermal radiation. This study clearly shows the parameterized effect, i.e. the Eckert number (Ec), the Prandtl number (Pr), the dynamic and thermal profiles along with the splice of the limited layer. A two-dimensional study of natural convection using nanoparticles $(\mathrm{Au})$ in a base fluid water was carried out in the Rayleigh rage $(103<\mathrm{Ra}<105)$ with the volume fraction $(0<\varphi<0.10)$. And considering the nature of the problem, the equations were solved digitally by Primo Ternik et al. [7]. Niu J et al. [8] Solved the heat transfer problem of a non-Newtonian fluid in a micro nano tube as a power series. This study shows the non-Newtonian property effect of the flow on the parameters like the dynamic profile, thermal, local Nusselt number of the rate of heat transfer. Kargar .A [9] dedicated his study to the analytical solution of natural convection of non-Newtonian fluid between two vertical plates by Homotopy (HPM) method. This study reveals the influence of the Prandtl and Eckert numbers on velocity and temperature profiles. Furthermore, a comparison was made between the numerical and analytical results that

This paper was recommended for publication in revised form by Regional Editor Bekir Yılbaş

${ }^{1}$ University of Skikda, El Hadaiek Road, B. O. 26, 21000 Skikda, Algeria

${ }^{2}$ University of Ouargla

*E-mail address: tabet21@yahoo.fr

Manuscript Received 04 February 2017, Accepted 22 April 2017 
were obtained. The work of A.A. Farooq [10] included a thorough comparison between the Runge-Kutta fourth order and the HPM methods; and the study reveals a range of applicability for HPM. Y. Rahmani [11], for his part, considered the presence of nanoparticles $\left(\mathrm{Ag}, \mathrm{Cu}, \mathrm{Al}_{2} \mathrm{O}_{3}\right.$, and $\left.\mathrm{TiO}_{2}\right)$ in the water as a base fluid and their influence on the heat coefficient, velocity and temperature distributions. The problem of natural convection flow of a nanofluid has been solved analytically and numerically by the HPM and Runge-Kutta methods respectively.

In recent decades, a new analytical methods appeared such as the method of variation of iterations (VIM) [12-13], reconstruction method of iterations changes (RVIM) [14], the spectral Homotopy method (SHPM) [15], the Adomian decomposition method (ADM) [16] and the Adomian Modified decomposition [17-18]. These methods provide a rapidly converging solution and require no discretization with a very high accuracy.

In this work, we study the natural convection in a non-Newtonian fluid / nanofluid between two vertical plates using three types of nanofluids namely Silver-water, Oxide Copper-Water and Oxid Titanium water. Strongly coupled nonlinear ordinary differential equations of the second order are proposed. They were solved numerically by the fourth order Runge-Kutta method, and analytically by a newe Adomian decomposition approach named the Adomian generalized method (GDM) walls [19].

\section{MATHEMATICAL FORMULATION}

Natural convection of a non-Newtonian fluid / nanofluid between two vertical flat plates travelling the distance $2 \mathrm{~b}$ ( Figure 1)

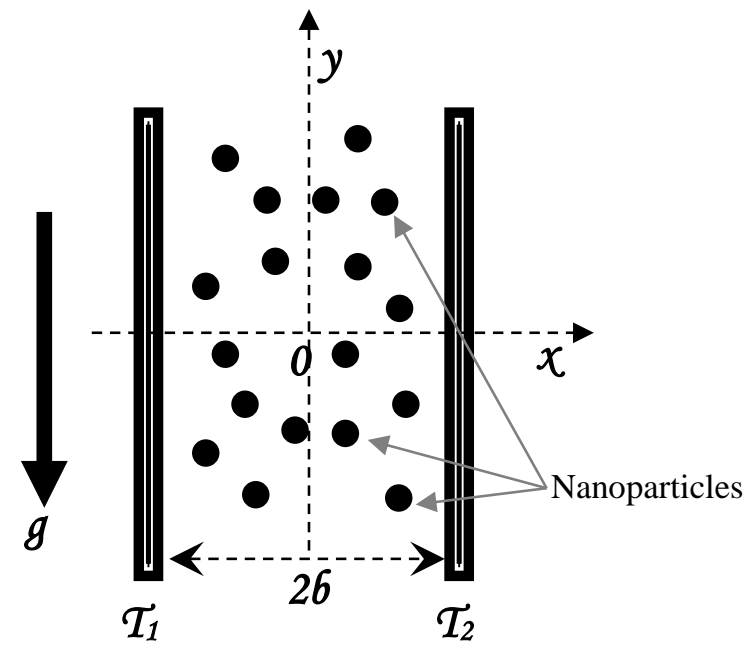

Figure 1. Flow geometry

It is assumed that the temperature on the walls is constant in $T_{1}$ and $T_{2}$ respectively.

The motion of an incompressible viscous nano-fluid in Cartesian coordinates is given as follows [22]:

$$
\mu_{n f} \frac{d^{2} v}{d x^{2}}+6 \beta^{3}\left(\frac{d v}{d x}\right)^{2} \frac{d^{2} v}{d x^{2}}+\rho_{n f} \delta\left(T-T_{m}\right) g=0
$$

In Cartesian coordinates, the energy equation of the natural convection for an incompressible nanofluid standing between two flat walls takes the following form:

$$
k_{n f} \frac{d^{2} T}{d x^{2}}+\mu_{n f}\left(\frac{d v}{d x}\right)^{2}+2 \beta^{3}\left(\frac{d v}{d x}\right)^{4}=0
$$

where

$\delta \quad$ the dimensionless non-Newtonian viscosity 
$\rho_{n f} \quad$ the nanofluid density $\mathrm{kg} / \mathrm{m}^{3}$

$\mu_{n f} \quad$ the dynamic viscosity of nanofluids $\mathrm{kg} / \mathrm{ms}$

$K_{n f} \quad$ the nanofluid thermal conductivity $w / m K$

$c_{p_{n f}}$ the specific heat of nanofluids at constant pressure $J / k g K$

The settings $\rho_{n f}, \mu_{n f}$ and $k_{n f}$ are expressed [20]:

$$
\begin{gathered}
\rho_{n f}=(1-\varphi) \cdot \rho_{f}+\psi \cdot \rho_{s} \\
\mu_{n f}=\frac{\mu_{f}}{(1-\varphi)^{2.5}} \\
\frac{K_{n f}}{K_{f}}=\frac{\left(k_{s}+2 k_{f}\right)-2 \varphi\left(k_{f}-k_{s}\right)}{\left(k_{s}+2 k_{f}\right)+2 \varphi\left(k_{f}-k_{s}\right)} \\
\left(\rho \cdot c_{p}\right)_{n f}=(1-\varphi) \cdot\left(\rho \cdot c_{p}\right)_{f}+\varphi \cdot\left(\rho \cdot c_{p}\right)_{s}
\end{gathered}
$$

where

$\varphi \quad$ the solid volume fraction

In a solid-liquid mixture (nano particles and base fluid), the volume fraction of the nanoparticles is defined as the solid volume of the nanoparticles Vs divided by the sum of the volumes of all components making up the nanofluid (liquid and solid). Therefore, the $\varphi$ volume fraction is given by

$$
\varphi=\frac{V_{\text {solide }}}{V_{\text {solide }}+V_{\text {liquide }}}
$$

For the investigated problem, it is very important to make the normalization of the equations; and for that purpose, we have to consider the dimensionless variables $V, \eta$ and $\theta$ defined by:

$$
\left\{\begin{array}{l}
V=\frac{v}{V_{0}} \\
\eta=\frac{x}{b} \text { Where }-1 \leq \eta \leq+1 \\
\theta=\frac{T-T_{m}}{T_{1}-T_{2}}
\end{array}\right.
$$

where

$V \quad$ Non-dimensional velocity or general nonlinear operator

$\theta \quad$ Non-dimensional temperature or general nonlinear operator

Taking into account the equation (3) and system (5), the equations (1 and 2) can also be written in the following form:

$$
\begin{gathered}
\frac{d^{2} V}{d \eta^{2}}+6 \delta(1-\varphi)^{2.5}\left(\frac{d V}{d \eta}\right)^{2} \frac{d^{2} V}{d \eta^{2}}+\theta=0 \\
\frac{d^{2} \theta}{d \eta^{2}}+E_{c} P_{r} \zeta(1-\varphi)^{-2.5}\left(\frac{d V}{d \eta}\right)^{2}+2 \delta E_{c} P_{r} \zeta\left(\frac{d V}{d \eta}\right)^{4}=0
\end{gathered}
$$


where dimensionless non-Newtonian viscosity $(\delta)$, Prandtl number (Pr), Eckert number (Ec), and the parameter forms:

$$
\begin{gathered}
\zeta=\frac{\left(k_{s}+2 k_{f}\right)+2 \varphi\left(k_{f}-k_{s}\right)}{\left(k_{s}+2 k_{f}\right)-2 \varphi\left(k_{f}-k_{s}\right)} \\
E_{c}=\frac{\rho_{f} \cdot V_{0}{ }^{2}}{c_{p_{f} \cdot\left(T_{1}-T_{2}\right)}} \\
P_{r}=\frac{\mu_{f} \cdot\left(\rho c_{p}\right)_{f}}{\rho_{f} \cdot k_{f}} \\
\delta=\frac{\beta V_{0}{ }^{2}}{\mu_{f} \cdot b^{2}}
\end{gathered}
$$

With the boundary conditions on the following fixed walls:

- dynamic conditions: $V( \pm 1)=0$

- thermal conditions: $\theta( \pm 1)= \pm 1 / 2$

The Skin friction coefficient $\boldsymbol{c}_{\boldsymbol{f}}$ and the rate of heat transfer $\mathrm{h}$ provide an indication on the physical shear wall and the heat transfer fluid, respectively. These coefficients are given by [21]:

$$
\left\{\begin{array}{l}
c_{f}=\left.\frac{d V}{d \eta}\right|_{\eta=-1}=V^{\prime}(-1) \\
h=\left.\frac{d \theta}{d \eta}\right|_{\eta=-1}=\theta^{\prime}(-1)
\end{array}\right.
$$

\section{THE THEORETICAL METHOD OF GDM}

The principle of this method is as follows:

Considering the following equation $[16,19]$ :

$$
F u(t)=g(t)
$$

where $\mathrm{F}$ is the ordinary and partial differential operator including linear and nonlinear terms. Equation (11) can also be written as follows:

$$
L u+R u+N u=g(t)
$$

The non- linear term $\mathrm{Nu}$ equation (12) can take the following form:

$$
N u=\sum_{n=0}^{+\infty} A_{n}\left(u_{0}, u_{1}, u_{2}, \ldots \ldots \ldots, u_{n}\right)
$$

$A_{n}$ where the term represents polynomials Adomian, which can be calculated by the following formula

$$
: A_{n}=\frac{1}{n !}\left[\frac{d^{n}}{d \lambda^{n}}\left[N\left(\sum_{n=0}^{\infty} \lambda^{i} u_{i}\right)\right]\right]_{\lambda=0}, n=0,1,2, \ldots \ldots, n
$$

The important part of the equation (11) is the nonlinear term $\mathrm{Nu}$. Indeed, since the Adomian method appeared, a number of researchers [17, 18] manifested interest in the development of new algorithms that improve the nonlinear behavior $N u$ term of equation (11), increasing thereby the effectiveness of the standard Adomian method. Among these new algorithms, we can find the one developed by Yong- Chang et al. [19]. This algorithm is a method of generalized Adomian (GDM). 
This method retains the general shape of the Adomian decomposition method but differs in the decomposition strategy for the nonlinear term in nonlinear equations. Indeed, this strategy uses all the information necessary for obtaining successive terms of the solution of the nonlinear term.

In the GDM method, the decomposition of polynomials is expressed according to the following formula:

$$
\left\{\begin{array}{c}
J_{0}=N\left(u_{0}\right) \\
J_{n}=N\left(u_{0}+u_{1}+\cdots+u_{n}\right)-N\left(u_{0}+u_{1}+\cdots+u_{n-1}\right), n \geq 1
\end{array}\right.
$$

Then, when the polynomials of the generalized Adomian method are known, the components of the solution $\mathrm{u}$ is given by:

$$
u_{0}=\omega+L^{-1} g, u_{n+1}=-L^{-1}\left(R u_{n}+J_{n}\right)
$$

where

$\omega \quad$ Is a constant

Finally, after a few iterations, the solution by GDM to equation (11) can be given by:

$$
u=u_{0}+u_{1}+u_{2}+u_{3}+\cdots+u_{n}
$$

\section{IMPLEMENTING GDM METHOD}

Velocity distribution was used to calculate temperatures, heat transfer coefficient and the coefficient of friction.

$$
\begin{gathered}
N V=-6 \delta(1-\varphi)^{2.5}\left(V^{\prime}\right)^{2} V^{\prime \prime}-\theta \\
L \theta=-E_{c} P_{r} \zeta(1-\varphi)^{-2.5}\left(V^{\prime}\right)^{2}-2 \delta E_{c} P_{r} \zeta\left(V^{\prime}\right)^{4}
\end{gathered}
$$

where: $\mathrm{L}$ is a differential operator such that:

$$
L=\frac{d^{2}}{d \eta^{2}}
$$

The inverse operator $L^{-1}$ of the differential operator $L$ is expressed by:

$$
L^{-1}=\iint \boldsymbol{\mathbf { a }} d \eta d \eta
$$

By applying equation (21) on the equations (18 and 19), we obtain:

$$
\begin{gathered}
V(\eta)=V(0)+V^{\prime}(0) \eta+L^{-1}(N V) \\
\theta(\eta)=\theta(0)+\theta^{\prime}(0) \eta+L^{-1}(N \theta)
\end{gathered}
$$

with,

$$
\begin{gathered}
N V=-6 \delta(1-\varphi)^{2.5}\left(V^{\prime}\right)^{2} V^{\prime \prime}-\theta \\
N \theta=-E_{c} P_{r} \zeta(1-\varphi)^{-2.5}\left(V^{\prime}\right)^{2}-2 \delta E_{c} P_{r} \zeta\left(V^{\prime}\right)^{4}
\end{gathered}
$$

Representing the nonlinear parts of equations (18-19). The constants $\mathrm{V}(0)$ and $\theta(0)$ depend on the boundary conditions $(9 a-b)$. In general, form the solutions take the following form: 
Journal of Thermal Engineering, Research Article, Vol. 4, No. 6, pp. 2496-2508, October, 2018

$$
\begin{aligned}
& V(n)=\sum_{\eta=0}^{\infty} V_{n}=V_{0}+L^{-1}(N V) \\
& \theta(n)=\sum_{\eta=0}^{\infty} \theta_{n}=\theta_{0}+L^{-1}(N \theta)
\end{aligned}
$$

where $V_{0}$ and $\theta_{0}$ are expressed by:

$$
\begin{gathered}
V_{0}=a \eta-\frac{b \eta^{3}}{6} \\
\theta_{0}=b \eta
\end{gathered}
$$

Thereafter, the application of the generalized Adomian algorithm (15) allows obtaining polynomials $\left\langle\left(A_{0}, B_{0}\right),\left(A_{1}, B_{1}\right), \ldots \ldots .\left(A_{n}, B_{n}\right)\right\rangle$ for the distributions of velocity and temperature. They are expressed as:

$$
\begin{gathered}
A_{0}=6 a^{2} b \delta \eta(1-\varphi)^{2.5}-6 a b^{2} \delta \eta^{3}(1-\varphi)^{2.5}+\frac{3}{2} b^{3} \delta \eta^{5}(1-\varphi)^{2.5} \\
B_{0}=-2 a^{4} \operatorname{EcPr} \delta \xi+4 a^{3} b \operatorname{EcPr} \delta \eta^{2} \xi-3 a^{2} b^{2} \operatorname{EcPr} \delta \eta^{4} \xi+a b^{3} \operatorname{EcPr} \delta \eta^{6} \xi- \\
\frac{1}{8} b^{4} \operatorname{EcPr} \delta \eta^{8} \xi-\frac{a^{2} \operatorname{EcPr} \xi}{(1-\varphi)^{2.5}}+\frac{1}{(1-\varphi)^{2.5}} a b \operatorname{EcPr} \eta^{2} \xi-\frac{1}{4(1-\varphi)^{2.5}} b^{2} \operatorname{EcPr} \eta^{4} \xi
\end{gathered}
$$

$$
\begin{gathered}
A_{1}=-6 a^{2} b \delta \eta(1-\varphi)^{2.5}+6 a b^{2} \delta \eta^{3}(1-\varphi)^{2.5}-\frac{3}{2} b^{3} \delta \eta^{5}(1-\varphi)^{2.5}-144 a^{8} b \delta^{4} \eta(1- \\
\varphi)^{10 .}+576 a^{7} b^{2} \delta^{4} \eta^{3}(1-\varphi)^{10 .}-1008 a^{6} b^{3} \delta^{4} \eta^{5}(1-\varphi)^{10 .}+1008 a^{5} b^{4} \delta^{4} \eta^{7}(1- \\
\varphi)^{10 .}-630 a^{4} b^{5} \delta^{4} \eta^{9}(1-\varphi)^{10 .}+252 a^{3} b^{6} \delta^{4} \eta^{11}(1-\varphi)^{10}-63 a^{2} b^{7} \delta^{4} \eta^{13}(1-\varphi)^{10}+ \\
9 a b^{8} \delta^{4} \eta^{15}(1-\varphi)^{10 .}-\frac{9}{16} b^{9} \delta^{4} \eta^{17}(1-\varphi)^{10}
\end{gathered}
$$

$B_{1}=2 a^{4} \operatorname{EcPr} \delta \xi-4 a^{3} b \operatorname{EcPr} \delta \eta^{2} \xi+3 a^{2} b^{2} \operatorname{EcPr} \delta \eta^{4} \xi-a b^{3} \operatorname{EcPr} \delta \eta^{6} \xi+\frac{1}{8} b^{4} \operatorname{EcPr} \delta \eta^{8} \xi+$ $\frac{a^{2} \operatorname{EcPr} \xi}{(1-\varphi)^{2.5}}-\frac{a b \operatorname{EcPr} \eta^{2} \xi}{(1-\varphi)^{2.5}}+\frac{b^{2} \mathrm{EcPr} \eta^{4} \xi}{4(1-\varphi)^{2.5}}-4 a^{6} \operatorname{EcPr} \delta^{2} \xi(1-\varphi)^{2.5}+12 a^{5} b \operatorname{Ec} \operatorname{Pr} \delta^{2} \eta^{2} \xi(1-\varphi)^{2.5}-$ $15 a^{4} b^{2} \operatorname{EcPr} \delta^{2} \eta^{4} \xi(1-\varphi)^{2.5}+10 a^{3} b^{3} \operatorname{EcPr} \delta^{2} \eta^{6} \xi(1-\varphi)^{2.5}-\frac{15}{4} a^{2} b^{4} \operatorname{EcPr} \delta^{2} \eta^{8} \xi(1-$ $\varphi)^{2.5}+\frac{3}{4} a b^{5} \operatorname{EcPr} \delta^{2} \eta^{10} \xi(1-\varphi)^{2.5}-\frac{1}{16} b^{6} \operatorname{Ec} \operatorname{Pr} \delta^{2} \eta^{12} \xi(1-\varphi)^{2.5}-32 a^{12} \operatorname{EcPr} \delta^{5} \xi(1-$ $\varphi)^{10 .}+192 a^{11} b \operatorname{Ec} \operatorname{Pr} \delta^{5} \eta^{2} \xi(1-\varphi)^{10 .}-528 a^{10} b^{2} \operatorname{EcPr} \delta^{5} \eta^{4} \xi(1-\varphi)^{10 .}+$ $880 a^{9} b^{3} \operatorname{Ec} \operatorname{Pr} \delta^{5} \eta^{6} \xi(1-\varphi)^{10 .}-990 a^{8} b^{4} \operatorname{Ec} \operatorname{Pr} \delta^{5} \eta^{8} \xi(1-\varphi)^{10 .}+$ $792 a^{7} b^{5} \operatorname{EcPr} \delta^{5} \eta^{10} \xi(1-\varphi)^{10 .}-462 a^{6} b^{6} \operatorname{EcPr} \delta^{5} \eta^{12} \xi(1-\varphi)^{10}+$ $198 a^{5} b^{7} \operatorname{EcPr} \delta^{5} \eta^{14} \xi(1-\varphi)^{10 .}-\frac{495}{8} a^{4} b^{8} \operatorname{EcPr} \delta^{5} \eta^{16} \xi(1-\varphi)^{10 .}+$ $\frac{55}{4} a^{3} b^{9} \operatorname{Ec} \operatorname{Pr} \delta^{5} \eta^{18} \xi(1-\varphi)^{10 .}-\frac{33}{16} a^{2} b^{10} \operatorname{EcPr} \delta^{5} \eta^{20} \xi(1-\varphi)^{10 .}+\frac{3}{16} a b^{11} \operatorname{EcPr} \delta^{5} \eta^{22} \xi(1-$ $\varphi)^{10 .}-\frac{1}{128} b^{12} \operatorname{EcPr} \delta^{5} \eta^{24} \xi(1-\varphi)^{10}$

The components of the solution of dynamic and thermal fields as GDM method are expressed by:

$$
V_{1}=\frac{1}{4} \delta\left(-8 a^{3} \eta+4 a^{2} b \eta^{3}-\frac{6}{5} a b^{2} \eta^{5}+\frac{b^{3} \eta^{7}}{7}\right)(1-\varphi)^{2.5}
$$




$$
\begin{aligned}
\theta_{1}=-\frac{1}{(1-\varphi)^{2.5}} & 0.125 \operatorname{Ec} \operatorname{Pr} \xi\left(4 . a^{2} \eta^{2}-0.666 a b \eta^{4}+0.066 b^{2} \eta^{6}+8 . a^{4} \delta \eta^{2}(1 .-\varphi)^{2.5}\right. \\
& -2.666 a^{3} b \delta \eta^{4}(1 .-\varphi)^{2.5}+0.8 a^{2} b^{2} \delta \eta^{6}(1 .-\varphi)^{2.5} \\
& \left.-0.142 a b^{3} \delta \eta^{8}(1 .-\varphi)^{2.5}+0.011 \delta \eta^{10}(1 .-\varphi)^{2.5}\right) \\
V_{2}=-\frac{3}{16} & b \delta\left(5.333 a^{2} \eta^{3}\left((1 .-\varphi)^{2.5}+24 . a^{6} \delta^{3}(1 .-\varphi)^{10 .}\right)-1.6 a b \eta^{5}\left((1 .-\varphi)^{2.5}\right.\right. \\
+ & \left.96 . a^{6} \delta^{3}(1 .-\varphi)^{10 .}\right)+0.19047619047619047 b^{2} \eta^{7}\left((1 .-\varphi)^{2.5}\right. \\
+ & \left.672 . a^{6} \delta^{3}(1 .-\varphi)^{10 .}\right)-74.66 a^{5} b^{3} \delta^{3} \eta^{9}(1 .-\varphi)^{10 .} \\
+ & 30.545454545454547 a^{4} b^{4} \delta^{3} \eta^{11}(1 .-\varphi)^{10 .} \\
& -8.615384615384617 a^{3} b^{5} \delta^{3} \eta^{13}(1 .-\varphi)^{10 .}+1.6 a^{2} b^{6} \delta^{3} \eta^{15}(1 .-\varphi)^{10 .} \\
- & 0.1764705882352941 a b^{7} \delta^{3} \eta^{17}(1 .-\varphi)^{10 .} \\
+ & \left.0.008771929824561403 b^{8} \delta^{3} \eta^{19}(1 .-\varphi)^{10 .}\right)
\end{aligned}
$$

$$
\begin{aligned}
\theta_{2}= & \frac{1}{128(1-\varphi)^{2.5}} \operatorname{Ec} \operatorname{Pr} \xi\left(-0.7272727272727272 a b^{5} \delta^{2} \eta^{12}\left((1 .-\varphi)^{5 .}\right.\right. \\
& \left.+1056 . a^{6} \delta^{3}(1 .-\varphi)^{12.5}\right)+0.04395604395604396 b^{6} \delta^{2} \eta^{14}\left((1 .-\varphi)^{5 .}\right. \\
& \left.+7392 . a^{6} \delta^{3}(1 .-\varphi)^{12.5}\right)-2.2857142857142856 a b^{3} \delta \eta^{8}\left(-1 .(1 .-\varphi)^{2.5}\right. \\
& \left.+10 . a^{2} \delta(1 .-\varphi)^{5 .}+880 . a^{8} \delta^{4}(1 .-\varphi)^{12.5}\right) \\
& +0.17777777777777778 b^{4} \delta \eta^{10}\left(-1 .(1 .-\varphi)^{2.5}+30 . a^{2} \delta(1 .-\varphi)^{5 .}\right. \\
& \left.+7920 \cdot a^{8} \delta^{4}(1 .-\varphi)^{12.5}\right)-1.066 b^{2} \eta^{6}\left(1 .+12 . a^{2} \delta(1 .-\varphi)^{2.5}\right. \\
& \left.-60 . a^{4} \delta^{2}(1 .-\varphi)^{5 .}-2112 . a^{10} \delta^{5}(1 .-\varphi)^{12.5}\right) \\
& +64 . a^{2} \eta^{2}\left(-1 .-2 . a^{2} \delta(1 .-\varphi)^{2.5}+4 . a^{4} \delta^{2}(1 .-\varphi)^{5 .}\right. \\
& \left.+32 . a^{10} \delta^{5}(1 .-\varphi)^{12.5}\right)-10.66 a b \eta^{4}\left(-1 .-4 . a^{2} \delta(1 .-\varphi)^{2.5}\right. \\
& \left.+12 . a^{4} \delta^{2}(1 .-\varphi)^{5 .}+192 . a^{10} \delta^{5}(1 .-\varphi)^{12.5}\right) \\
& -105.6 a^{5} b^{7} \delta^{5} \eta^{16}(1 .-\varphi)^{12.5} \\
& +25.882352941176467 a^{4} b^{8} \delta^{5} \eta^{18}(1 .-\varphi)^{12.5} \\
& -4.631578947368421 a^{3} b^{9} \delta^{5} \eta^{20}(1 .-\varphi)^{12.5} \\
& +0.5714285714285714 a^{2} b^{10} \delta^{5} \eta^{22}(1 .-\varphi)^{12.5} \\
& -0.043478260869565216 a b^{11} \delta^{5} \eta^{24}(1 .-\varphi)^{12.5} \\
& \left.+0.0015384615384615387 b^{12} \delta^{5} \eta^{26}(1 .-\varphi)^{12.5}\right)
\end{aligned}
$$

And finally, after a number of iterations, the solutions to equations $(6,7)$ by the GDM method are given by:

$$
\begin{aligned}
& V=V_{0}+V_{1}+V_{2}+V_{3}+\cdots \ldots .+V_{n} \\
& \theta=\theta_{0}+\theta_{1}+\theta_{2}+\theta_{3}+\cdots \ldots .+\theta_{n}
\end{aligned}
$$

\section{RESULTS AND DISCUSSIONS}

The Runge-Kutta method of order four and analytically by a new generalized Adomian technique (GDM), solved the nonlinear problem of the natural convection flow of a non-Newtonian fluid/nanofluid between two vertical planes walls, governed by equations $(6,7)$ numerically. This work was carried out on different types of nanoparticles and water as a base fluid. The thermo-physical properties of these nanoparticles are shown in Table 1. 
Table 1. Thermo-physical properties of nanofluids

\begin{tabular}{|c|c|c|c|}
\hline & $\rho\left(\mathrm{Kg} / \mathrm{m}^{3}\right)$ & $\mathrm{Cp}\left(\mathrm{J} / \mathrm{Kg} .{ }^{\circ}\right)$ & $\mathrm{K}\left(\mathrm{W} / \mathrm{m} .{ }^{\circ} \mathrm{K}\right)$ \\
\hline Water & 997.1 & 4179 & 0.613 \\
\hline $\mathrm{Ag}$ & 10500 & 235 & 429 \\
\hline $\mathrm{Cuo}$ & 535.6 & 6500 & 20 \\
\hline $\mathrm{Tio}_{2}$ & 4250 & 686.2 & 8.9538 \\
\hline
\end{tabular}

Figures (2-3) and Table 2 show a comparison between the numerical and analytical results, and the adopted analytical technique is shown to be more effective. A comparison with other studies [11, 22] was made, as illustrated in histograms (1-2), and table 3 for $\delta=0.5, E c=1 \operatorname{Pr}=1$ and $. \varphi=0$.

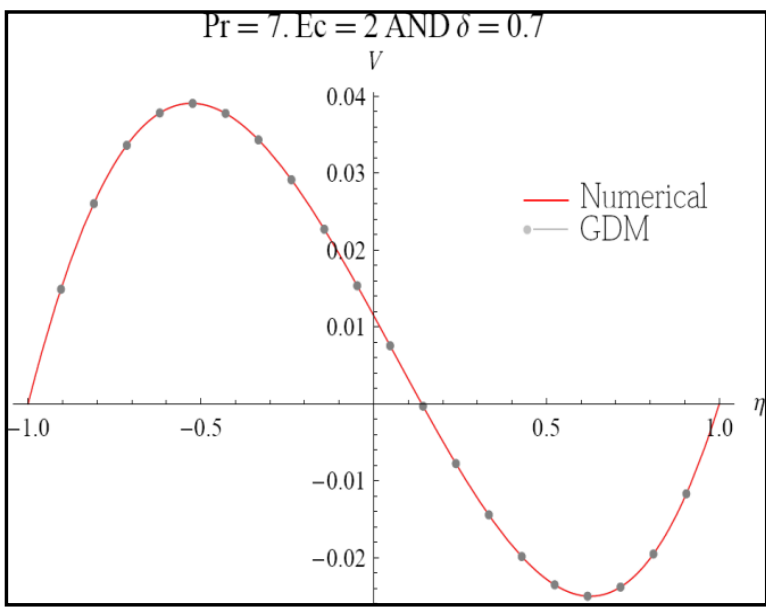

Figure 2. Dimensional profile of velocity distribution (numerical and analytical results) when: $\delta=0.7, E c=2 \operatorname{Pr}=7$ and $\varphi=0$.

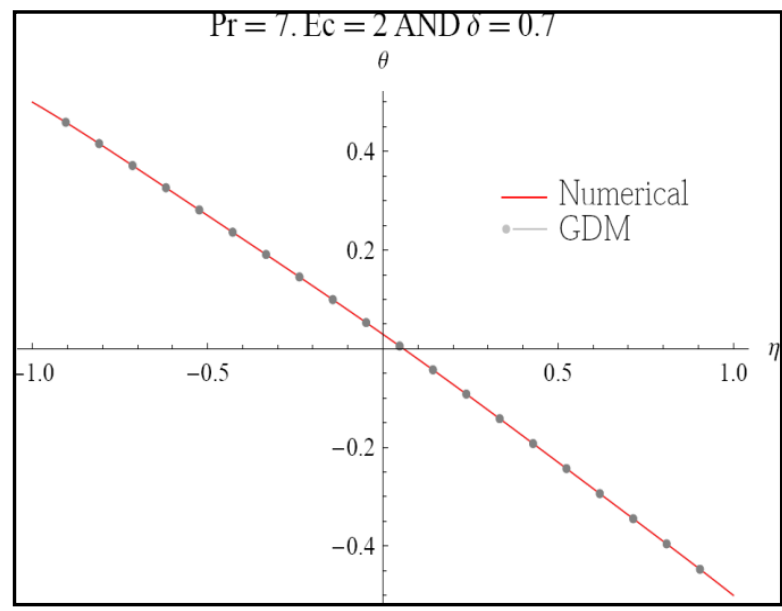

Figure 3. Dimensional profile of temperatures distribution (numerical and analytical results) when: $\delta=0.7, E c=2 \operatorname{Pr}=7$ and $\varphi=0$.

Table 2. Comparison between the numerical and analytical results of the velocity and temperature distribution when: $\delta=0.7, E c=2$ and $\operatorname{Pr}=7$.

\begin{tabular}{|c|c|c|c|c|c|c|c|}
\hline$\eta$ & $V_{\text {Num }}$ & $V_{G D M}$ & $\left|V_{\text {Num }}-V_{G D M}\right|$ & $\eta$ & $\theta_{\text {Num }}$ & $\theta_{G D M}$ & $\left|\theta_{\text {Num }}-\theta_{G D M}\right|$ \\
\hline-1.0 & 0.0000000 & 0.0000000 & 0.0000000 & -1.0 & +0.5 & 0.500000 & 0.0000000 \\
\hline-0.75 & 0.0312291 & 0.0312292 & 0,0000001 & -0.75 & 0.388349 & 0.388347 & 0,000002 \\
\hline-0.50 & 0.0389874 & 0.0389873 & 0,0000001 & -0.50 & 0.270220 & 0.270224 & 0,000004 \\
\hline-0.25 & 0.0299131 & 0.0299130 & 0,0000001 & -0.25 & 0.151397 & 0.151391 & 0,000006 \\
\hline 0.00 & 0.0115104 & 0.0115106 & 0,0000002 & 0.00 & 0.029454 & 0.029458 & 0,000004 \\
\hline 0.25 & -0.0086613 & -0.0086611 & 0,0000002 & 0.25 & -0.098109 & -0.098108 & 0,000001 \\
\hline 0.50 & -0.0227898 & -0.0227897 & 0,0000001 & 0.50 & -0.230249 & -0.230247 & 0,000002 \\
\hline 0.75 & -0.0225941 & -0.0225942 & 0,0000001 & 0.75 & -0.363705 & -0.363703 & 0,000002 \\
\hline 1.00 & 0.0000000 & 0.0000000 & 0.0000000 & 1.00 & -0.5 & -0.500000 & 0.0000000 \\
\hline
\end{tabular}




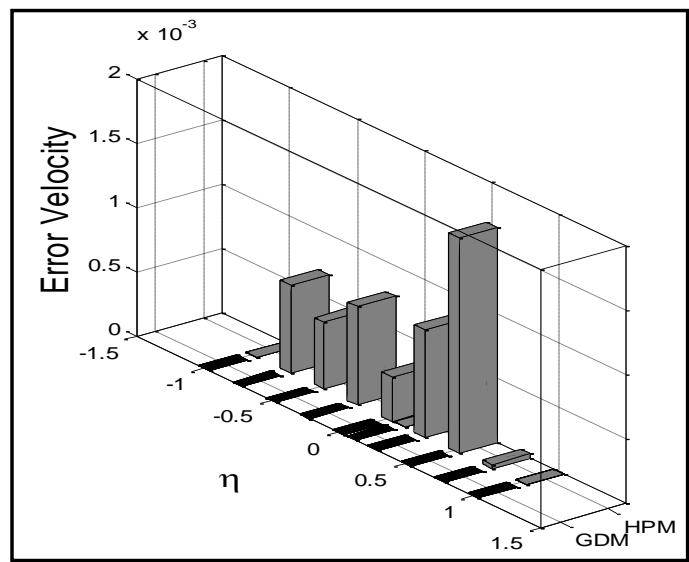

Histogram 1. Comparison of errors between the adopted analytical technique and other works [11] for velocity distribution when $\delta=0.5, E c=1 \operatorname{Pr}=1$ and $. \varphi=0$

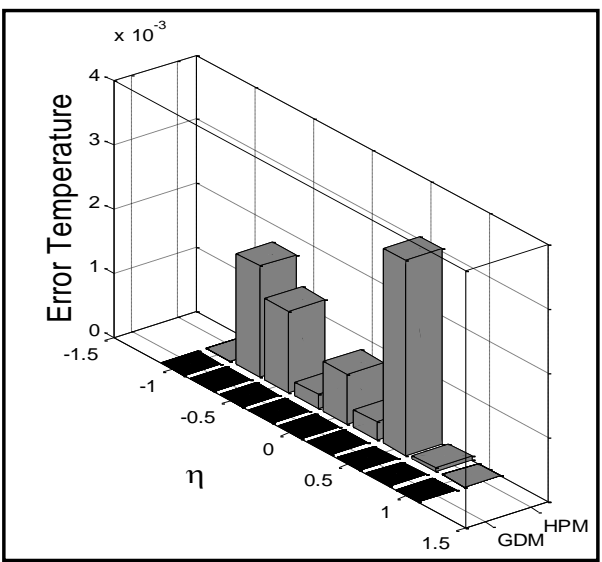

Histogram 2. Comparison of errors between the analytical technique used and other work [11] for temperature distribution when $\delta=0.5, E c=$ $1 \operatorname{Pr}=1$ and $. \varphi=0$

Table 3. Comparison of the analytical technique used in other works [22] for velocity and temperature distributions when: $\delta=E c=\operatorname{Pr}=1$ and $\varphi=0.01$.

\begin{tabular}{|c|c|c|c|c|c|c|c|c|}
\hline & $V(\eta)^{\text {Num }}$ & $V(\eta)^{D T M}[22]$ & $V(\eta)^{L S M}[22]$ & $V(\eta)^{G D M}$ & $\theta(\eta)^{\text {Num }}$ & $\theta(\eta)^{D T M}[22]$ & $\theta(\eta)^{L S M}[22]$ & $\theta(\eta)^{G D M}$ \\
\hline-1.00 & 0.000000000 & $-10^{-11}$ & $3 * 10^{-12}$ & 0.000000000 & 0.50 & 0.50 & 0.50 & 0.50 \\
\hline-0.90 & 0.013888702 & 0.01402428 & 0.0137141 & 0.013888851 & 0.45043148 & 0.45059686 & 0.450000 & 0.45043148 \\
\hline-0.80 & 0.023620556 & 0.02368182 & 0.0232664 & 0.02362048 & 0.40071897 & 0.40113389 & 0.400000 & 0.40071457 \\
\hline-0.70 & 0.029489465 & 0.02945437 & 0.0290255 & 0.029489203 & 0.35094362 & 0.35161056 & 0.350000 & 0.35094234 \\
\hline-0.60 & 0.031888322 & 0.03182368 & 0.0313930 & 0.031888487 & 0.30114965 & 0.30202640 & 0.300000 & 0.30114217 \\
\hline-0.50 & 0.031279886 & 0.03127150 & 0.0307996 & 0.031279147 & 0.25135346 & 0.25238090 & 0.250000 & 0.25135964 \\
\hline-0.40 & 0.028164333 & 0.02827959 & 0.0277008 & 0.028164248 & 0.20155266 & 0.20267356 & 0.200000 & 0.20155324 \\
\hline-0.30 & 0.023053394 & 0.02332968 & 0.0225728 & 0.023053238 & 0.15173412 & 0.15290389 & 0.150000 & 0.15173125 \\
\hline-0.20 & 0.016454874 & 0.01690353 & 0.0159085 & 0.016454781 & 0.10188071 & 0.10307139 & 0.100000 & 0.10188089 \\
\hline-0.10 & 0.008866496 & 0.00948289 & 0.0082130 & 0.008866207 & 0.05197647 & 0.05317557 & 0.050000 & 0.05197384 \\
\hline 0.00 & 0.000776279 & 0.00154951 & 0.0000001 & 0.000776327 & 0.002010267 & 0.00321593 & 0.0000 & 0.002010351 \\
\hline 0.10 & -0.007333249 & -0.00641485 & -0.0082121 & -0.007333345 & -0.04802206 & -0.04680801 & -0.050000 & -0.04802570 \\
\hline 0.20 & -0.014979179 & -0.01392846 & -0.0159072 & -0.014979278 & -0.09811697 & -0.09689681 & -0.100000 & -0.09811541 \\
\hline 0.30 & -0.021672314 & -0.02050956 & -0.0225753 & -0.021672598 & -0.14826378 & -0.14705093 & -0.150000 & -0.14826124 \\
\hline 0.40 & -0.026912911 & -0.02567640 & -0.0277042 & -0.026912781 & -0.19844679 & -0.19727080 & -0.200000 & -0.19844581 \\
\hline 0.50 & -0.030190188 & -0.02894723 & -0.0307938 & -0.030190327 & -0.24864875 & -0.24755700 & -0.250000 & -0.24864712 \\
\hline 0.60 & -0.030988255 & -0.02984030 & -0.0313923 & -0.030988357 & -0.29885599 & -0.29791001 & -0.300000 & -0.29885412 \\
\hline 0.70 & -0.028801308 & -0.02787389 & -0.0290255 & -0.028801217 & -0.34906512 & -0.34833031 & -0.350000 & -0.34906745 \\
\hline 0.80 & -0.023159274 & -0.02256617 & -0.0232664 & -0.023159784 & -0.39929111 & -0.39881842 & -0.400000 & -0.39929217 \\
\hline 0.90 & -0.013660270 & -0.01343546 & -0.0137142 & -0.013660278 & -0.44957637 & -0.44937485 & -0.450000 & -0.44957637 \\
\hline 1.00 & 0.00 & $-2 * 10^{-11}$ & $-3 * 10^{-12}$ & $-5 * 10^{-24}$ & -0.50 & -0.50 & -0.50 & -0.50 \\
\hline
\end{tabular}

Figures (4-5) show the effect of volume fraction $\varphi$ of the nanoparticles on velocity and temperature distributions for Silver-water nanofluid $\delta=1, \mathrm{Ec}=2$ and $\operatorname{Pr}=7$.

We can clearly see in Figure (4) that there is a proportional relationship between velocity distribution and volume fraction $\varphi$ against temperature distribution (Figure 5). As it can be observed, the temperature decreases with increasing volume fraction $\varphi$. 


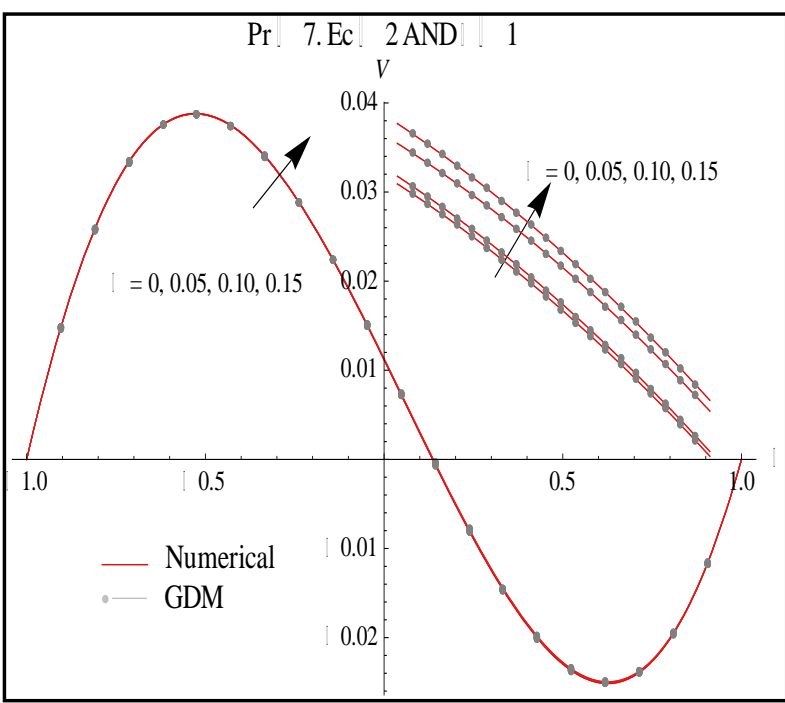

Figure 4. Effect of $\varphi$ volume fraction on velocity profile when $\delta=1, E c=2$ and $\operatorname{Pr}=7$. (case of silver , Ag).

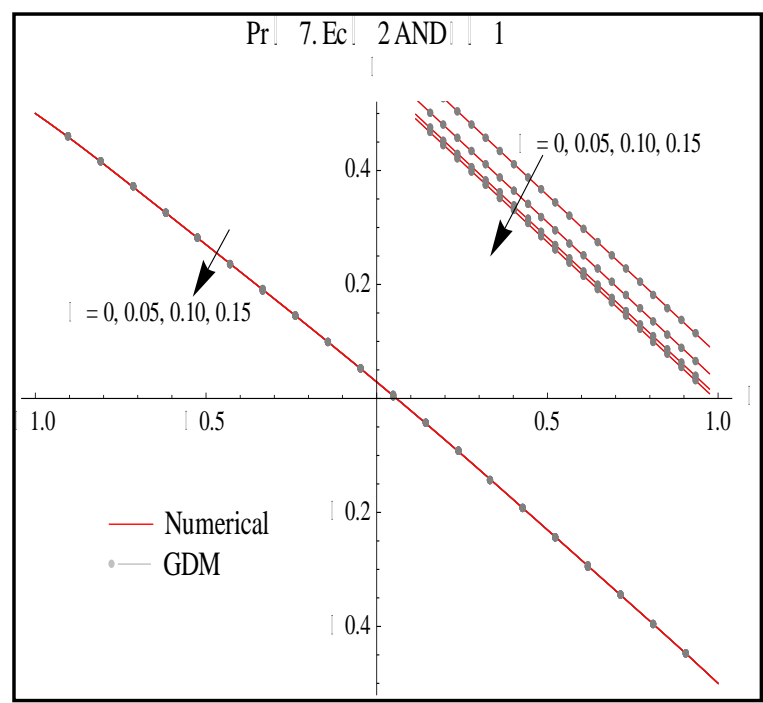

Figure 5. Effect of $\varphi$ volume fraction on temperature profile when $\delta=1, E c=2$ and $\operatorname{Pr}=7$. (case of silver, Ag).

The effect of the Eckert number on velocity and temperature profiles for the nanofluid (water as a base fluid) is illustrated in Figures ( 6 and 7), when $\delta=1, \varphi=0.09$ and $\operatorname{Pr}=7$.

From these figures, One can clearly see that increasing the Eckert number causes an increase in velocity and temperature; velocity takes a maximum value in the vicinity of $\eta=-0.5$, whereas the lowest velocity is observed at the point $\eta= \pm 1$.

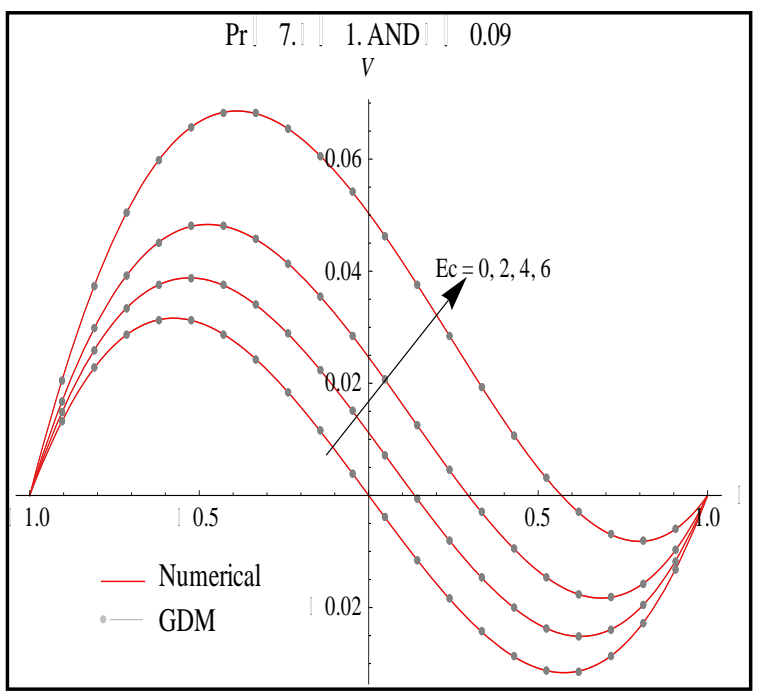

Figure 6. Effect of Eckert number Ec on the velocity profile when $\delta=1, \varphi=0.09$ and $\operatorname{Pr}=7$. (case silver, Ag).

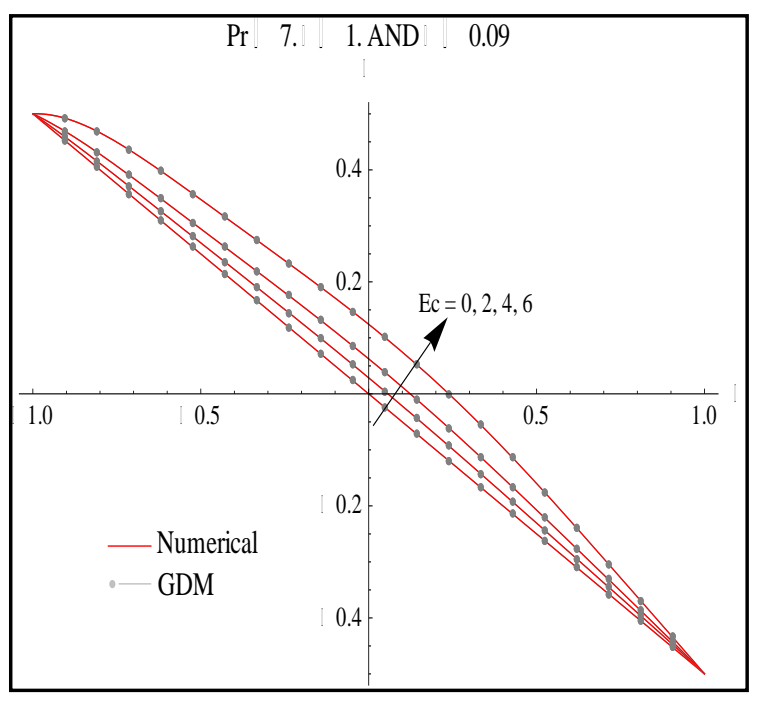

Figure 7. Effect of Eckert number Ec on the temperature profile when $\delta=1, \varphi=0.09$ and $\operatorname{Pr}=$ 7.(case of silver, Ag).

Figures (8 and 9) show the influence of non-Newtonian viscous parameter $\delta$ of the dynamic and thermal profiles for silver-water nanofluid, when $\mathrm{Ec}=5, \varphi=0.12$ and $\operatorname{Pr}=7$.

It is observed that the velocity and temperature distributions decrease with the increase of the nonNewtonian viscous parameter $\delta$, and the highest velocity is manifested in the neighborhood of $\eta=-0.5$ in the case at which the non-Newtonian viscous parameter $\delta$ is zero 


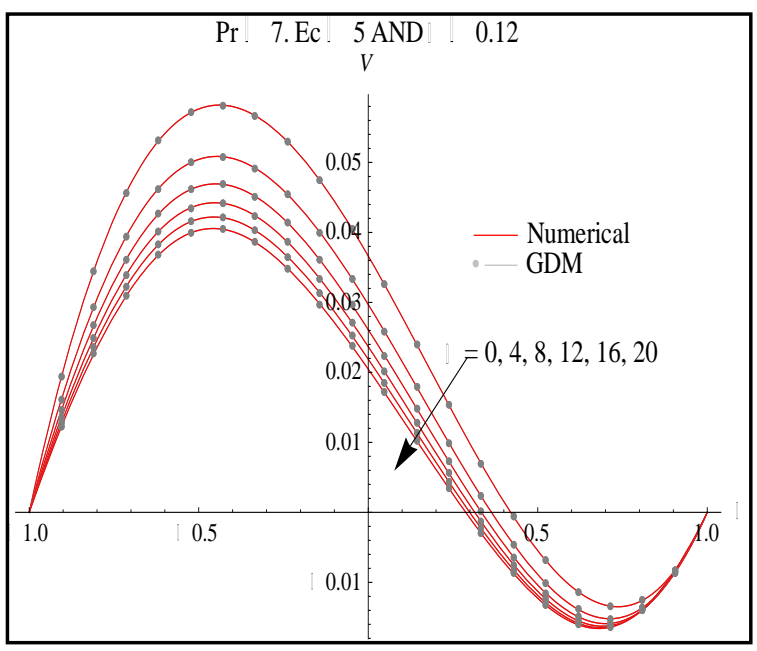

Figure 8. Effect of the non-Newtonian viscous parameter $\delta$ on the velocity profile when $E c=$ 5, $\varphi=0.12$ and $\operatorname{Pr}=7$. (case of silver, $\mathrm{Ag}$ ).

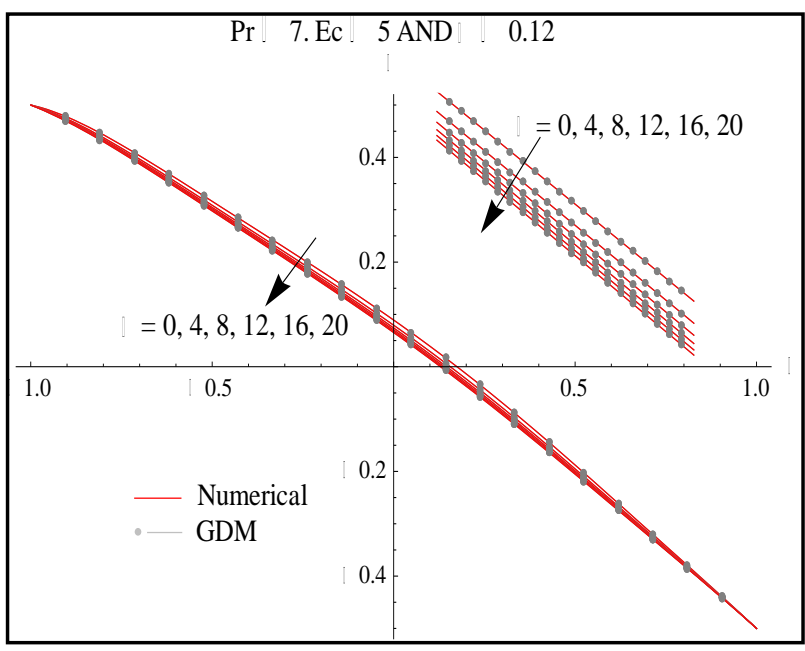

Figure 9. Effect of non-Newtonian viscous $\delta$ parameter on the temperature profile when $E c=5, \varphi=$ 0.12 and $\operatorname{Pr}=7$.(case of silver, Ag).

In what follows, we investigate the effects of volume fraction $\varphi$ and the nature of the nanoparticles on the friction and heat transfer coefficients as shown in Figures (10-11).

These figures reveal clearly that the friction coefficient and the heat transfer coefficient h for $\mathrm{TiO}_{2}$ water nanofluid are higher compared to those of other nanofluids (Ag-water $\mathrm{CuO}$-water), and are strongly bound to thermo-physical properties and the nature of the nanoparticles.

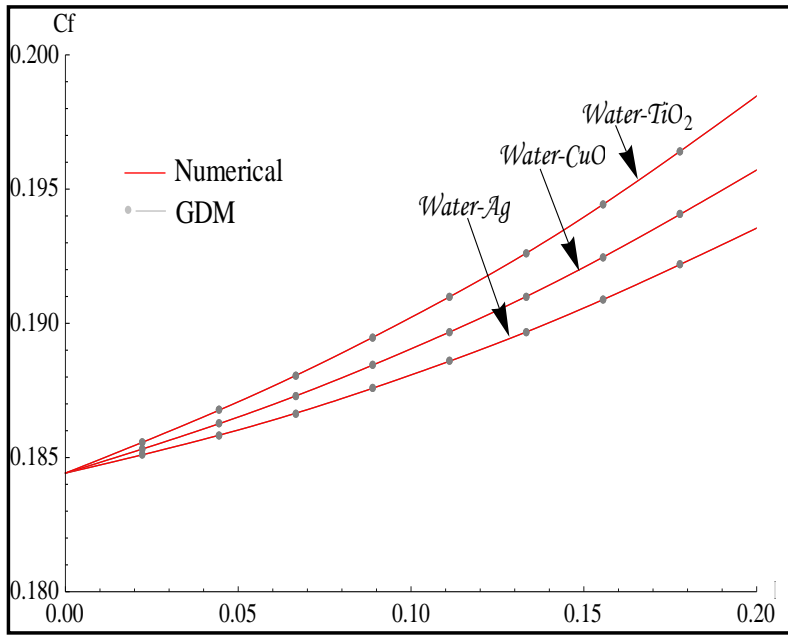

Figure 10. Effect of the nature of nanofluids on the friction coefficient $c_{f}$ when $\delta=2, E c=4 \operatorname{andPr}=7$.

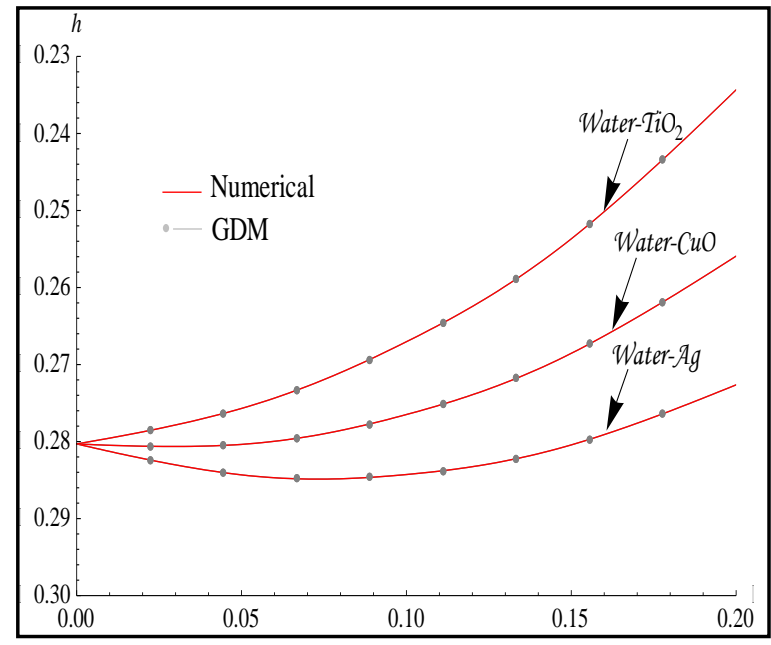

Figure 11. Effect of the nature of nanofluids on the heat transfer coefficient $\mathrm{h}$ when $\delta=2, E c=$ 4 and $\operatorname{Pr}=7$

\section{CONCLUSION}

In this article, Dynamic and thermal contribution to the flow of a non-Newtonian fluid / nanofluid between two nano-infinite vertical planar walls, was mathematically formulated. Subsequently, a set of strongly coupled nonlinear differential equations of the second order governing velocity and temperature distributions was treated numerically and analytically. In fact, the numerical solution was obtained by the Runge-Kutta technique of fourth order, by contrast, the analytical solution was provided by a new generalized Adomian technique (GDM).

The main conclusions we can draw from this study are: 
The presence of nanoparticles $\left(\mathrm{Ag}, \mathrm{CuO}\right.$ and $\left.\mathrm{TiO}_{2}\right)$ in water, used as a base fluid, substantially increases the coefficient of friction and the characteristics of heat transfer. Under these conditions, the thermal conductivity of the nanofluid is clearly higher than that of the base fluid.

The biggest advantage of the analytical methods compared to digital processing methods, resides in calculation time;indeed, the solution by the analytical methods is given in the form of rapidly converging series whereas numerical methods require a specific adaptation of differential equations to the algorithms besides, they need programming in a scientific language, and take a large computational time, depending on to the desired accuracy.

Comparing the generalized Adomian decomposition technique (GDM) relative to other works (HPM) shows the advantages of the adopted technique (GDM) in terms of precision and velocity of convergence.

\section{REFERENCE}

[1] Ostrach S. (1972). Natural convection in enclosures. Advances in heat transfer.,8,161-227.

[2] Khalifa A.J. (2001). Natural convective heat transfer coefficient-A review II, surfaces in two and threedimensional enclosures. Energy conversion and management., 42,505-517.

[3] Jha B.K., Ajibade A.O. (2010). Transient natural convection flow between vertical parallel plates: one plate isothermally heated and the other thermally insulated. Journal of process mechanical engineering, 224(4)247252.

[4] Noghrehabadi A., Behseresht A., Ghalambaz M. (2013). Natural convection of nanofluid over vertical plate embedded in porous medium: prescribed surface heat flux. Applied mathematics and mechanics.,1573-2754.

[5] Rashad, A. M., El-Hakiem, M. A., Abdou, M. M. M. (2011). Natural convection boundary layer of a nonNewtonian fluid about a permeable vertical cone embedded in a porous medium saturated with a nanofluid. Computers \& Mathematics with Applications, 62(8), 3140-3151.

[6] Narahar M. (2009). Natural convection in unsteady Couette flow between two vertical parallel plates in the presence of constant heat flux and radiation. MACMESE'09 Proceedings of the 11th WSEAS international conference on mathematical and computational methods in science and engineering, 73-78.

[7] Ternik, P., Rudolf, R., Zunic, Z. (2012). Numerical study of heat transfer enhancement of homogeneous water-Au nanofluid under natural convection. Materials and Technology, 46(3), 257-261.

[8] Niu, J., Fu, C., \& Tan, W. (2012). Slip-flow and heat transfer of a non-Newtonian nanofluid in a microtube. PLoS One, 7(5), e37274.

[9] Kargar, A., Akbarzade, M. (2012). Analytic solution of natural convection flow of a non-newtonian fluid between two vertical flat plates using homotopy perturbation method (HPM). World Applied Sciences Journal, 20(11), 1459-1465.

[10] Farooq, A. A., Siddiqui, A. M., Rana, M. A., Haroon, T. (2012). Application of He's Method in Solving a System of Nonlinear Coupled Equations Arising in Non-Newtonian Fluid Mechanics. International Journal of Applied Mathematical Research, 1, 130-140.

[11] Rahmani, Y., Yousefi, R., Ghasemi, S. E., Ganji, D. D. (2013). Thermal and fluid effects of nonNewtonian water-based nanofluids on the free convection flow between two vertical planes. Phys. Rev. Res. Int, 3(4), 688-701.

[12] He, J. H., Wu, X. H. (2007). Variational iteration method: vew development and applications, Computers and Mathematics with applications, 54, 881-894.

[13] He, J. H. (2007). Variational iteration method-Some recent results and new interpretations, Journal of Computational and applied mathematics., 207, 3-17.

[14] Imani, A. A., Rostamian, Y., Ganji, D. D., \& Rokni, H. B. (2012). Analytical Investigation of JefferyHamel Flow with High Magnetic Field and Nano Particle by RVIM.

[15] Khidir, A. A. (2014). Spectral-Homotopy Perturbation Method for Solving Governing MHD JefferyHamel Problem. Journal of Computational Methods in Physics, 2014.

[16] Adomian, G. (1994). Solving Frontier Problems of Physics: The Decomposition Method, Dodrecht. Kluwer Academic Publishers.

[17] Song, L., Wang, W. (2013). A new improved Adomian decomposition method and its application to fractional differential equations. Applied Mathematical Modelling, 37(3), 1590-1598. 
[18] Hasan, Y. Q., Zhu, L. M. (2008). Modified Adomian decomposition method for singular initial value problems in the second-order ordinary differential equations. Surveys in Mathematics and its Applications, 3, 183-193.

[19] Jiao, Y. C., Dang, C., Yamamoto, Y. (2008). An extension of the decomposition method for solving nonlinear equations and its convergence. Computers \& Mathematics with Applications, 55(4), 760-775.

[20] Khanafer, K., Vafai, K., Lightstone, M. (2003). Buoyancy-driven heat transfer enhancement in a twodimensional enclosure utilizing nanofluids. International journal of heat and mass transfer, 46(19), 3639-3653.

[21] Rajagopal, K. R., Na, T. Y. (1985). Natural convection flow of a non-Newtonian fluid between two vertical flat plates. Acta Mechanica, 54(3-4), 239-246.

[22] Hatami, M., Ganji, D. D. (2014). Natural convection of sodium alginate (SA) non-Newtonian nanofluid flow between two vertical flat plates by analytical and numerical methods. Case Studies in Thermal Engineering, 2, 14-22.

[23] Kundu, B. (2015). Semianalytical methods for heat and fluid flow between two parallel plates. Journal of Thermal Engineering, 1(3), 175-181. 